\title{
A Novel Global Tractography Algorithm Based on an Adaptive Spin Glass Model
}

\author{
Pierre Fillard, Cyril Poupon, and Jean-François Mangin \\ LNAO - CEA/DSV/I²BM/Neurospin, Paris, France
}

\begin{abstract}
This paper introduces a novel framework for global diffusion MRI tractography inspired from a spin glass model. The entire white matter fascicle map is parameterized by pieces of fibers called spins. Spins are encouraged to move and rotate to align with the main fiber directions, and to assemble into longer chains of low curvature. Moreover, they have the ability to adapt their quantity in regions where the spin concentration is not sufficient to correctly model the data. The optimal spin glass configuration is retrieved by an iterative minimization procedure, where chains are finally assimilated to fibers. As a result, all brain fibers appear as growing simultaneously until they merge with other fibers or reach the domain boundaries. In case of an ambiguity within a region like a crossing, the contribution of all neighboring fibers is used determine the correct neural pathway. This framework is tested on a MR phantom representing a $45^{\circ}$ crossing and a real brain dataset. Notably, the framework was able to retrieve the triple crossing between the callosal fibers, the corticospinal tract and the arcuate fasciculus.
\end{abstract}

\section{Introduction}

The emergence of diffusion MRI for the past two decades enabled the in vivo study of anatomical connectivity via white matter tractography. Deterministic tractography algorithms reconstruct putative fascicles incrementally by following the main fiber directions as revealed by diffusion models (e.g, tensor or Q-Ball). However, such approach is prone to local errors in the estimate of the fiber directions (caused by noise and partial voluming) and may deviate from the true neural pathway. Probabilistic tractography [1/2 3] appeared as a way to handle the uncertainty of the fiber orientations by sampling each direction from a probability density function related to the diffusion model, and by repeating several thousands of times this technique from a seed point. While those methods was shown to be less noise-sensitive compared to their deterministic counterparts, their output is also very different: they return a connectivity map where each voxel is proportional to the probability of being connected to the seed. This raises the question of the statistical significance of those maps (which value is significant, which is not?). Moreover, further decomposition of fibers into morphological descriptors (e.g., length, curvature) for shape analysis becomes difficult. Recently, global alternatives to tractography were developed 45. In those, 
the entire neural pathway is the parameter to be optimized, which elegantly adds robustness to deterministic tractography.

In this work, we propose a novel framework for global tractography which is a complete overhaul of the spin glass model introduced in 6] The entire set of white matter fibers is parameterized by small segments called spins. Contrary to 6. (where spin positions and number are fixed), spins are allowed to move, rotate and duplicate. To control their degrees of freedom, spins are endowed with three potential energies: a diffusion, an interaction, and a generative potential. The diffusion potential attracts spins towards the main fiber directions, while the interaction potential encourages them to form longer chains of minimal curvature. The generative potential prevents a spin chain to end inside the domain by allowing the creation of new spins. The optimal spin configuration is finally retrieved by a global minimization procedure. As a result, all brain fibers appear as growing simultaneously until they merge with other fibers or reach the white matter boundaries. The advantages of such approach are fourfold: 1) it only relies on the two generally admitted priors that brain fibers have a low curvature and do not end inside white matter, 2) it does not require an estimation of the number nor directions of the fiber compartments in each voxel, 3) it automatically adapts the number of parameters (i.e., spins) to the data, and 4) it can be adapted to any type of diffusion model.

The rest of the paper is organized as follows. The framework for spin glass tractography (SGT) is exposed in Sec. 2. Experiments on real datasets are conducted in Sec. 3, before concluding in Sec. 4.

\section{An Adaptive Spin Glass Model for Tractography}

A spin $s$ is an oriented particle defined by its position $x$ and orientation $\vec{v}$ (of unit length). A spin glass $S$ is an ensemble of $N$ spins contained within a closed domain $\Omega: S=\left\{s_{i}\left(x_{i}, \vec{v}_{i}\right) / x_{i} \in \Omega\right\}_{0<i<N}$. A spin can be interpreted as a piece of fiber (point plus tangent), while $\Omega$ is the definition domain of the fibers.

Spins are endowed with three types of potential energy: a diffusion potential, an interaction potential and a generative potential. The diffusion potential acts as a non-stationary magnetic field attracting the spin orientations towards the main fiber directions. The interaction potential controls how spin associate with neighbors and embeds the prior that fibers have a low curvature. The generative potential authorizes the spontaneous generation of new spins to ensure that fibers do not end inside white matter. Note that the term "adaptive spin glass" is due to this last potential, which adapts the quantity of spins to the data. Fibers are finally retrieved by minimizing the sum of those three potentials over all spins in the glass. In the following, we first introduce each potential and present the algorithm for fiber reconstruction based on this spin glass model.

${ }^{1}$ We keep the terminology "spin glass" although we do not rigorously follow the concepts of the original spin glass model developed in statistical physics. 


\subsection{The Diffusion Potential}

Within our framework, the minima of the diffusion potential $E_{d}$ should coincide with the main fiber directions. The expression of this potential obviously depends on the diffusion model chosen to represent the data. However, we may follow this principle for a general definition. When the probability of finding a fiber in a direction is high, the potential should be low and conversely. Furthermore, when the probability is close to zero, the potential should tend towards infinity as it is very unlikely to find a fiber in that direction. Thus, $E_{d}$ should be proportional to the log-likelihood of the fiber presence in a direction. One can write, for any model:

$$
E_{d}(x, \vec{v})=-\log (p(x, \vec{v})),
$$

where $p(x, \vec{v})$ is the probability of existence of a fiber at location $x$ and in direction $\vec{v}$. Assuming that the main tensor eigenvectors are good estimates of the fiber directions, we provide the following expression of $p$ for a tensor field $D(x)$ : $p(x, \vec{v})=\vec{v}^{\top} D(x) \vec{v}\left(D(x)\right.$ should be normalized to ensure $\int_{S^{2}} p(x, \vec{v}) d v=1$, $S^{2}$ being the unit sphere). Dedicated expressions can be derived for other models. In particular, the orientation distribution functions (ODF) 7] are appealing since they directly provide the probability $p$. Note that multiple maxima are possible: in this case, the diffusion potential will act as a multi-modal magnetic field and attract a spin towards one particular fiber direction depending on its initial orientation.

\subsection{The Interaction Potential}

The interaction potential $E_{\text {int }}$ controls how spins interact with each others and encourages them to assemble into longer chains. Following [6], this potential embeds the prior that brain fibers have a low curvature. Basically, a spin orientation separates the spin glass into two sets: those which are behind it (backward spins) and those in front of it (forward spins). We define the set of forward (resp. backward) neighbors as $N^{f}(s)=\left\{s_{i} \in S /\left\|x_{i}-x\right\|<r\right.$ and $\left.\vec{v} \cdot \overrightarrow{x x_{i}}>0\right\}$ (resp. $N^{b}(s)=\left\{s_{i} \in S /\left\|x_{i}-x\right\|<r\right.$ and $\left.\left.\vec{v} \cdot \overrightarrow{x x_{i}} \leq 0\right\}\right) . r$ is the radius controlling the size of the neighborhood. A spin $s$ associates with a unique backward $s^{b}\left(x^{b}, \vec{v}^{b}\right)$ and forward $s^{f}\left(x^{f}, \vec{v}^{f}\right)$ neighbor such that the interaction potential of their trajectory $s^{b}-s-s^{f}$ is minimal.

We propose to define the interaction potential between a spin and its backward and forward neighbors as:

$$
\mathrm{E}_{i n t}\left(s, s^{b}, s^{f}\right)=-\frac{1}{5} \sum_{j=1}^{5} \log \left(\frac{\cos \alpha_{j}-\cos \alpha_{\max }}{1-\cos \alpha_{\max }}\right),
$$

where $\alpha_{1}$ (resp. $\alpha_{2,3,4,5}$ ) is defined as the angle between $\vec{v}^{b}$ and $x-x^{b}$ (resp. $x-x^{b}$ and $\vec{v}, \vec{v}$ and $x^{f}-x, x^{f}-x$ and $\vec{v}^{f}, \vec{v}^{b}$ and $\vec{v}^{f}$ ) (see Fig. 11). $\alpha_{\max }$ is the maximum angular deviation allowed and lie within the range $] 0, \pi / 2]$. Any angular difference greater than $\alpha_{\max }$ will produce an infinite potential which 


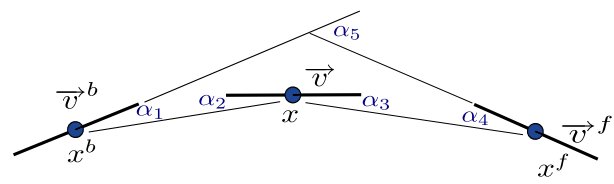

Fig. 1. Angular differences between a spin $s$ and its backward $\left(s^{b}\right)$ and forward $\left(s^{f}\right)$ neighbors. A spin is represented by a blue circle (position), and a black line (orientation). The role of $\alpha_{5}$ is to prevent the creation of a junction between two incompatible neighbors, which would result in an angular deviation greater than $\alpha_{\max }$.

forbids the spin association. Conversely, spins perfectly aligned will associate as their interaction potential is null. If a spin leads outside the glass, i.e., $x \pm \vec{v} \notin \Omega$, then no interaction potential is added for this half-neighborhood.

\subsection{The Generative Potential}

This last potential plays a particular role: it ensures that brain fibers do not end inside white matter, which can be translated by spin chains do not stop within the glass. There are situations where a spin cannot find a candidate neighbor to associate with, which causes a chain to end prematurely. This is the case when all neighbors have already been associated (we recall that a spin associates to a unique backward and forward neighbor), or when available neighbors lead to an infinite interaction potential (angular differences greater than $\alpha_{\max }$ ). In this case, the generative potential will become infinite, ordering the creation of a new spin to complete the broken chain. We formulate this potential as follows:

$$
\begin{aligned}
E_{\text {gen }}(s) & =\infty \text { if } \forall\left(s^{b}, s^{f}\right) \in N^{b}(s) \times N^{f}(s), E_{\text {int }}\left(s, s^{b}, s^{f}\right)=\infty \\
& =0 \text { otherwise }
\end{aligned}
$$

\subsection{Global Potential and Algorithm Overview}

The combination of the interaction (Eq. 11) and diffusion potentials (Eq. 21) has a competitive influence on the spin orientations and localizations. Hence, the optimal spin glass configuration corresponds to a trade-off between fidelity to the diffusion data and low curvature. The generative potential (Eq. 3) only affects the quantity of spins so that chains do not stop within the glass. The optimal spin configuration $S^{*}$ is obtained when the sum over all spins of the three potentials is minimal, i.e.:

$$
S^{*}=\min _{S=\left\{s_{i}\right\}} \sum_{i=1}^{N} E_{d}\left(s_{i}\right)+\lambda E_{\text {int }}\left(s_{i}, s_{i}^{b}, s_{i}^{f}\right)+E_{g e n}\left(s_{i}\right),
$$

where $\lambda$ controls the trade-off between interaction and diffusion potentials.

The optimal configuration is retrieved via an alternate minimization procedure. For a given spin glass configuration, spin positions and orientations are optimized to minimize their diffusion and interaction potentials only. Once the 

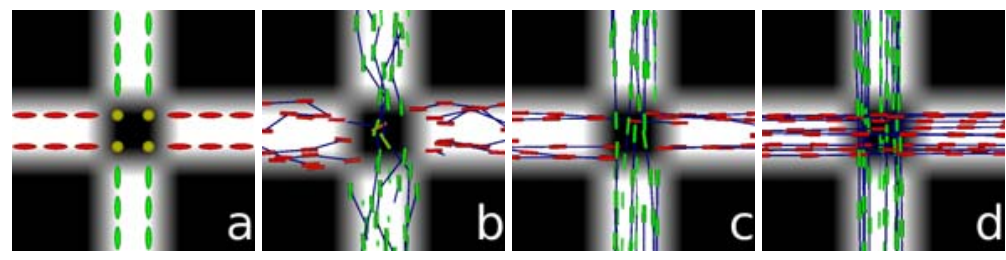

Fig. 2. Example of SGT of a synthetic fiber crossing. a) The tensor field. b) The initial spin glass: spins (represented by green and red cylinders) were randomly placed and aligned with the main tensor eigenvectors. The blue links represent the spin associations. c) The minimal energetic configuration of the spin glass in b). d) After convergence: spin chains have grown and merged to reconstruct the crossing area.

stable spin configuration is found, the generative potential is minimized by merging together compatible spin chains and by adding new spins at the extremities of remaining broken chains. During this phase, the contribution of all neighboring spin chains in regions of crossing fibers is used to determine the most likely pathways (the ones with the lowest possible curvature). Thus, there is no need for knowing the number of fiber compartments in each voxel, as those are automatically determined by the neighborhood. Finally, the process is reiterated until convergence (no more spin association and generation). Depending on the size of the dataset, from 10 to 100 iterations are necessary and tens to millions of spins are required. Example of SGT of a synthetic crossing is shown in Fig. 2 top row (20 iterations, 230 initial spins, 314 spins after convergence, $\lambda=1.0$, $\alpha_{\max }=45^{\circ}, r=3 \mathrm{~mm}$ ). In the next section, we evaluate the performance of the SGT algorithm on real datasets.

\section{Experiments}

We evaluated the spin glass algorithm on two datasets: a MR phantom consisting of a $45^{\circ}$ crossing and a real brain dataset. For each dataset, we compared the output of the SGT algorithm to a tensor-based streamline tractography (TBT) (the tensorlines [8]) and a Q-Ball-based streamline tractography (QBT) [9] (generalization of classical streamline techniques to Q-Ball) algorithms. For tensor fitting, we used the Rician noise removal strategy developed in [10]. For Q-Ball estimation, we opted for the spherical harmonics decomposition of 9] which provides an analytical formulation of the ODF. Note that the diffusion potential of the SGT was derived from the ODF and not the tensor. Parameters of the spin glass algorithm are: $\lambda=1, \alpha_{\max }=45^{\circ}$ and $r=3 \mathrm{~mm}$. The same angular threshold was used for the TBT and QBT to obtain comparable results.

\subsection{MR Phantom}

The crossing phantom was elaborated with hydrophobic fibers strongly tightened with a medium and immersed in a solution of water doped with gadolinium [1]. Acquisitions were done on a $1.5 \mathrm{~T}$ MRI scanner (4000 directions, b-value of 

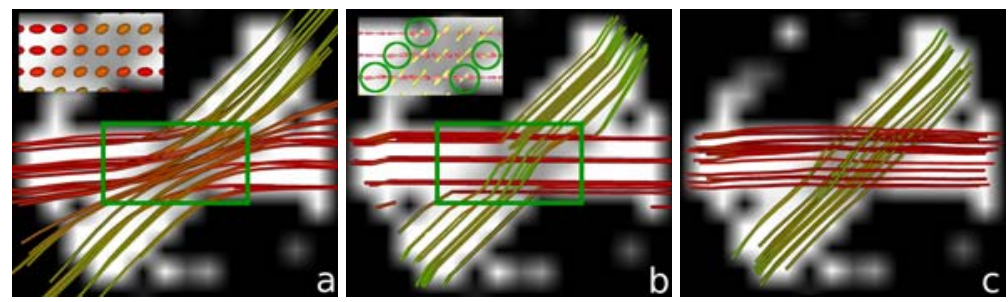

Fig. 3. SGT of the $45^{\circ}$ crossing MR phantom. a) Result of TBT. Tensors contained within the green rectangle are displayed. b) Result of QBT. ODF contained within the green rectangle are displayed (those circled in greens had only one maxima out of two detected). c) Result of SGT. The same ODF were used for QBT and SGT.

2000s. $\mathrm{mm}^{-2}$, image size: $32 \times 32 \times 3$, resolution: $\left.1 \times 1 \times 1.4 \mathrm{~mm}\right)$. Results of tractography are shown in Fig. 3. Obviously, TBT was not able to recover a crossing of this low angular difference, since tensors are unable to model the two-fiber compartment inside the crossing area. QBT algorithm was able to recover part of the crossing but deviates in regions where the ODF peaks are not well defined (mainly due to noise and partial voluming). Indeed, maxima are generally detected by thresholding the ODF not to extract small noisy peaks. In our case, the ODF circled in green (Fig. 3 b) had only one maxima detected out of the two expected, which caused several erroneous pathways. SGT, however, was able to pass the crossing by using the neighborhood to infer the most likely pathways (Fig. 3 c).

\subsection{Real Brain Dataset}

The brain dataset was acquired on a $1.5 \mathrm{~T}$ scanner with two protocols. First, a DT-MRI dataset of 41 directions and a b-value of $700 \mathrm{~s} . \mathrm{mm}^{-2}$ was acquired for TBT. Second, a HARDI dataset of 200 directions with a b-value of $3000 \mathrm{~s} . \mathrm{mm}^{-2}$ was acquired for QBT and SGT. Image size is $128 \times 128 \times 60$, resolution is: $1.8 \times$ $1.8 \times 2 \mathrm{~mm}$. Results of trackings are presented in Fig. 4 . For clarity reasons, only fibers passing through a sagittal slice of the corpus callosum (CC) are presented. Fibers are colored depending on the $\mathrm{CC}$ position they traverse (linear color gradient from the posterior in red to the anterior in blue of the CC). Nearly all fibers reconstructed by the TBT algorithm were redirected vertically because of the projection fibers (corona radiata) crossing the callosal fibers. QBT performed slightly better and fibers connecting the lateral part of the frontal and parietal lobes were found, showing that QBT is able to pass the corona radiata. However, the quality of the ODF was not good enough to allow QBT to recover the full set of association fibers crossing the CC. SGT was able to resolve this crossing area and exhibits many more interhemispheric connections. Moreover, the consistency of the coloring scheme indicates that the position of a fiber connecting the cortex is related to its anteroposterior position in the CC. SGT required 2 million spins to reconstruct the entire brain and three days of computations on a regular PC.

To further illustrate the performance of the SGT, we display in Fig. 5 the intersection between the CC, the corticospinal tract (CST), the arcuate 


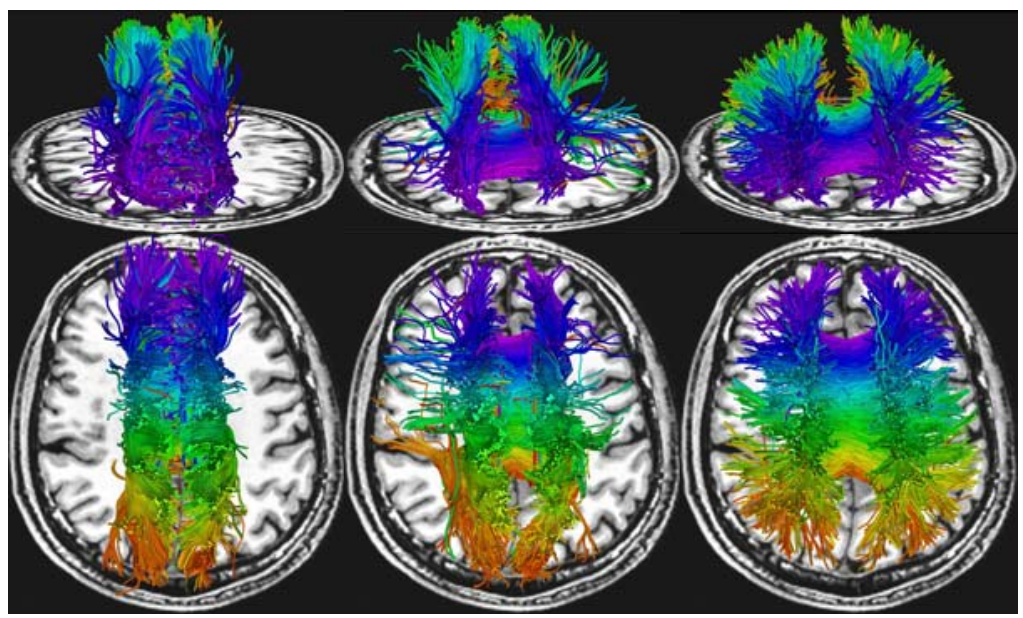

Fig. 4. Reconstruction of callosal fibers with three methods. Left: TBT. Middle: QBT. Right: SGT. All fibers of TBT are redirected vertically because of the surrounding corona radiata. QBT performed slightly better but missed a large part of the callosal fibers. SGT, by using the neighborhood to determine the most plausible pathways, was able to recover the myriad of fibers passing the corona radiata.

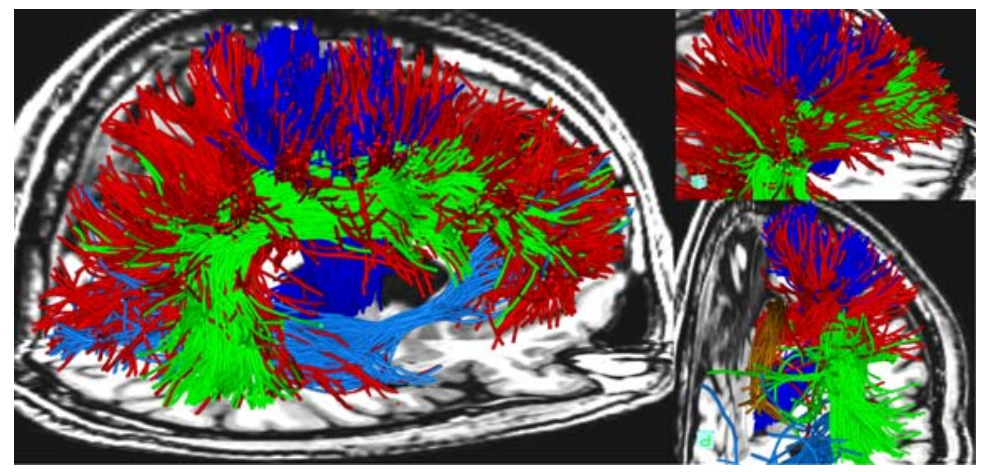

Fig. 5. Intersection between the CC (red), the CST (dark blue), the AF (green), the $\mathrm{CB}$ (orange) and the ILF (light blue) revealed by spin glass tractography. This region is one of the most complex crossing area accessible at this resolution of diffusion images.

fasciculus $(\mathrm{AF})$, the cingulum bundle $(\mathrm{CB})$, and the inferior longitudinal fasciculus (ILF). The triple-crossing region made by the CC, the CST and the AF was successfully reconstructed by the SGT algorithm.

\section{Conclusion}

In this work, we presented a new methodology for white matter fiber reconstruction inspired from a spin glass model. Spins are fiber elements endowed with a 
diffusion, interaction and generative potentials. The combination of those three potentials allows the creation of long chains of spins of low curvature and fitting the diffusion data at best, which grow until they merge with other chains or reach the domain boundaries. In case of an ambiguity (like a crossing), the pathways leading to the lowest curvature are automatically chosen. Such approach has the advantage not to require a prior knowledge on the number of fiber compartments per voxel, and can be adapted to any type of diffusion model. Experiments on a synthetic MR phantom and a real brain dataset indicated that such a global approach is more successful than deterministic streamline tensor or q-ball-based methods to recover crossing fibers. Notably, we showed that spin glass tractography can successfully reconstruct the triple crossing between the corpus callosum, the corticospinal tract and the arcuate fasciculus, which is one of the most complex crossing area accessible at this level of image resolution.

\section{References}

1. Parker, G., Alexander, D.: Probabilistic anatomic connectivity derived from the microscopic persistent angular structure of cerebral tissue. Philosophical Transactions of the Royal Society B 360, 893-902 (2005)

2. Friman, O., Farneback, G., Westin, C.F.: A bayesian approach for stochastic white matter tractography. IEEE TMI 25(8), 965-978 (2006)

3. Jbabdi, S., Woolrich, M., Andersson, J., Behrens, T.: A bayesian framework for global tractography. NeuroImage 37, 116-129 (2007)

4. Zhang, F., Hancock, E.R., Goodlett, C., Gerig, G.: Probabilistic white matter fiber tracking using particle filtering and von Mises-Fisher sampling. MedIA 13(1), 5-18 (2009)

5. Kreher, B., Madeer, I., Kiselev, V.: Gibbs tracking: A novel approach for the reconstruction of neuronal pathways. MRM 60, 953-963 (2008)

6. Mangin, J.F., Poupon, C., Cointepas, Y., Riviére, D., Papadopoulos-Orfanos, D., Clark, C.A., Régis, J., Le Bihan, D.: A framework based on spin glass models for the inference of anatomical connectivity from diffusion-weighted MR data. NMR in Biomedicine 15, 481-492 (2002)

7. Tuch, D.: Q-ball imaging. MRM 52(6), 1358-1372 (2004)

8. Weinstein, D.M., Kindlmann, G.L., Lundberg, E.C.: Tensorlines: Advectiondiffusion based propagation through diffusion tensor fields. In: IEEE Vis 1999, pp. 249-253 (1999)

9. Descoteaux, M., Deriche, R., Knösche, T., Anwander, A.: Deterministic and probabilistic tractography based on complex fibre orientation distribution TMI 28(2) (2009)

10. Fillard, P., Arsigny, V., Pennec, X., Ayache, N.: Clinical DT-MRI estimation, smoothing and fiber tracking with log-Euclidean metrics. TMI 26, 1472-1482 (2007)

11. Poupon, C., Rieul, B., Kezele, I., Perrin, M., Poupon, F., Mangin, J.F.: New diffusion phantoms dedicated to the study and validation of HARDI models. MRM 60, 1276-1283 (2008) 